\title{
ANALYSIS OF A MULTI-SPOKE OPTION FOR THE RIA DRIVER LINAC*
}

\author{
D. Gorelov ${ }^{\dagger}$, T. Grimm, W. Hartung, F. Marti, X. Wu, and R.C. York \\ National Superconducting Cyclotron Laboratory, MSU, East Lansing, MI 48824, USA
}

\begin{abstract}
Beam dynamics simulations of the proposed Rare Isotope Accelerator (RIA) [1] driver linac have been done. The RIA driver linac is designed to accelerate stable ion beams from proton to uranium to final energies of 400 $\mathrm{MeV} / \mathrm{u}$ for the heaviest and about $900 \mathrm{MeV} / \mathrm{u}$ for the lightest ions with beam powers of 100 to $400 \mathrm{~kW}$. Two stripping sections are used to increase the charge state of heavy ions and minimize the total accelerating voltage required. To achieve the final beam power and to reduce the ion source requirements, multi-charge state beam acceleration is used. Multi-spoke structures [2] in the high-energy part of the driver linac have been proposed as an alternative to the baseline design of 6-cell elliptical structures [3]. A comparative analysis of this alternative is explored including beam dynamics, error constraints, and manufacturing issues.
\end{abstract}

\section{INTRODUCTION}

The baseline RIA driver linac design uses $805 \mathrm{MHz}$ 6cell elliptical cavities with geometric $\beta_{g}$ of $0.47,0.61$ and 0.81 to accelerate beam from the $2^{\text {nd }}$ charge-stripping chicane at $\sim 85 \mathrm{MeV} / \mathrm{u}$ to final energies of $\geq 400 \mathrm{MeV} / \mathrm{u}$ [3]. (An alternative design without the $\beta_{g}=0.81$ cavities is also being evaluated, but would not affect these results.) Figure 1 shows a section view of a $\beta_{g}=0.47,805 \mathrm{MHz} 6$ cell elliptical cavity. A prototype $\beta_{g}=0.47,805 \mathrm{MHz} 6-$ cell elliptical cavity was completed and tested in 2002, exceeding design goals [4].

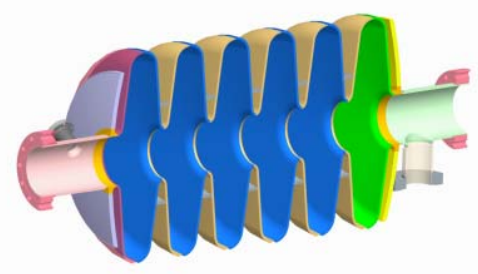

Figure 1: $\beta_{g}=0.47805 \mathrm{MHz}$ 6-cell elliptical cavity.

An option of using $322 \mathrm{MHz}$ triple-spoke cavities in lieu of the $805 \mathrm{MHz}$ 6-cell elliptical cavities was proposed in 2002 [2]. Because of the lower operating frequency of the triple-spoke structures, the required number of cavities in the driver linac for the spoke cavity option will be smaller. Two types of triple-spoke cavities are required, with optimum $\beta=0.48$ and $\beta=0.62$. Figure 2 shows a section view of a $322 \mathrm{MHz}$, triple-spoke cavity for $\beta=0.62$. No prototype has been made of this cavity.

\footnotetext{
*Work supported by MSU and NSF PHY 0110253.
}

†'gorelov@nscl.msu.edu

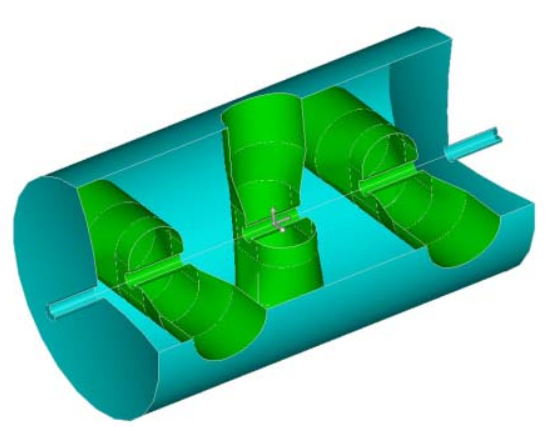

Figure 2: $\beta=0.62322 \mathrm{MHz}$ triple-spoke cavity.

Longitudinal and transverse beam dynamics studies were performed to compare these two alternative designs in the high- $\beta$ section of the RIA driver linac using the computer codes LANA [5] and DIMAD [6].

\section{LONGITUDINAL BEAM DYNAMICS}

The longitudinal acceptance of the elliptical structure linac with average reference particle phase in the accelerating gaps $\varphi_{\mathrm{s}} \sim-30^{\circ}$ is comparable in size with the longitudinal acceptance of a triple-spoke structure linac with $\varphi_{s} \sim-15^{\circ}$, as shown in Figure 3. For the 6-cell elliptical cavities an accelerating gradient of $E_{0} T=8.28 \mathrm{MV} / \mathrm{m}$ was used, corresponding to a peak electric field of $E_{p}=27.5 \mathrm{MV} / \mathrm{m}$ and requiring 192 cavities. For the triple-spoke structures, $E_{0} T=7 \mathrm{MV} / \mathrm{m}$ was used, corresponding to a peak electric field of $E_{p}=20 \mathrm{MV} / \mathrm{m}$ and requiring 152 triple-spoke cavities for $\varphi_{\mathrm{s}} \sim-15^{\circ}$. (Note that a 6-cell elliptical cavity system with a frequency of $644 \mathrm{MHz}$ ( $8^{\text {th }}$ harmonic of initial frequency) would require 154 cavities, but, like the triple-spoke, this cavity has never been prototyped.)

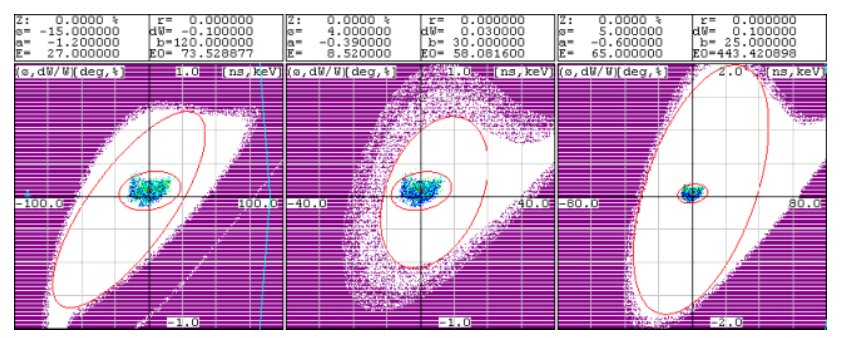

Figure 3: Longitudinal beam acceptances for 6-cell elliptical cavities with $\varphi_{\mathrm{s}} \sim-30^{\circ}$ (left), triple-spoke cavities with $\varphi_{\mathrm{s}}=-15^{\circ}$ (middle), and $\varphi_{\mathrm{s}}=-30^{\circ}$ (right, doubled scale). A multi-charge longitudinal beam emittance of $\sim 9 \pi \mathrm{keV} / \mathrm{u}$ nsec is shown for comparison.

\section{TRANSVERSE BEAM DYNAMICS}

Three transverse focusing lattices were established. The rf defocusing from both cavity types was found to be 
similar, with strength an order of magnitude smaller than that of the lattice focusing elements. As a consequence, the transverse beam dynamics are predominantly determined by the focusing elements and the lattice layout and not the cavity choice.

The cryostat dimensions for all three focusing lattices are based on the rectangular cryomodule design with cryogenic alignment rail proposed for RIA at Michigan State University [7]. A prototype cryomodule for the elliptical cavities with geometric $\beta_{g}=0.47$ is under construction and easily extends to the other cavity types.

\section{Solenoid Focusing Lattice}

The layout of a solenoid focusing lattice is shown in Figure 4, with each cryostat containing four cavities and a superconducting solenoid magnet for transverse focusing. The effective lengths of the solenoids range from 0.5 to $1.25 \mathrm{~m}$, with a maximum magnetic field of about $9 \mathrm{~T}$. The transverse phase advance of each cryostat is about $90^{\circ}$. Two extra solenoids are necessary to match the beam condition from the $2^{\text {nd }}$ charge-stripping chicane to a periodic solenoid focusing lattice. Figure 5 shows the beam envelopes for ${ }^{238} \mathrm{U}$ with three charge states, $87+$ to $89+$. The initial beam emittance for all charge states was assumed to be $2.9 \pi \mathrm{mm}$ mrad. The maximum beam size for all three charge states is about $8.6 \mathrm{~mm}$.

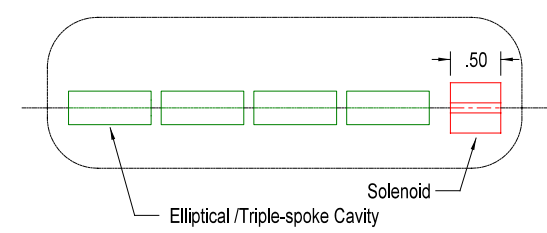

Figure 4: Layout of solenoid focusing lattice.

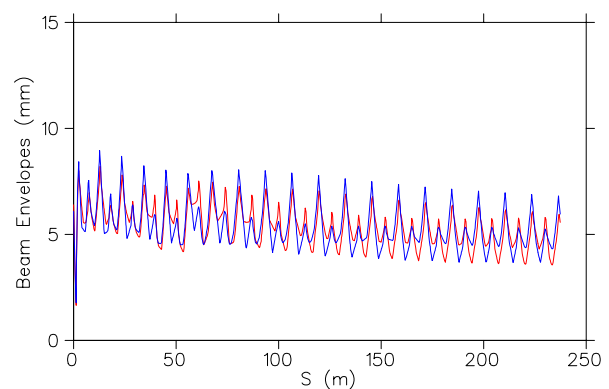

Figure 5: Multi-charge beam envelopes of ${ }^{238} \mathrm{U}$ in the solenoid focusing lattice with Triple-spoke cavities.

\section{Two-Quadrupole Focusing Lattice}

In this energy range, quadrupoles can provide a more efficient and less costly focusing system than superconducting solenoids. A layout with two superconducting quadrupoles per cryostat is shown in Figure 6. The effective lengths of the quadrupole magnets are $0.25 \mathrm{~m}$ with an aperture of $5 \mathrm{~cm}$. For a $90^{\circ}$ transverse phase advance per cryostat, the maximum pole face magnetic field is about $0.5 \mathrm{~T}$. A short section with four quadrupole magnets is required to match the beam from the $2^{\text {nd }}$ charge-stripping chicane to the regular focusing lattice. Figure 7 shows the beam envelopes for the same beam as Figure 5. The maximum beam size for all the three charge states is about $11.3 \mathrm{~mm}$ significantly larger than that of the solenoid focusing lattice. The focusing properties of this lattice are virtually identical to those of a previously presented doublet-focusing lattice $[3,8]$.

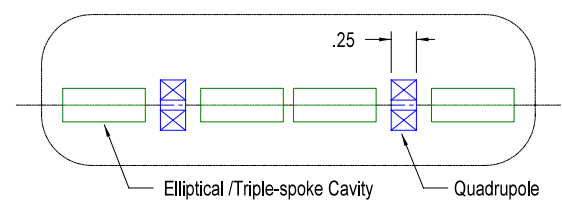

Figure 6: Layout of the two-quadrupole focusing lattice.

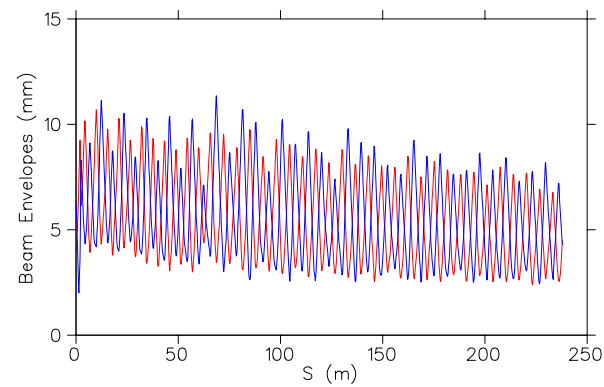

Figure 7: Multi-charge beam envelopes of ${ }^{238} \mathrm{U}$ in the twoquadrupole focusing lattice with Triple-spoke cavities.

\section{Five-Quadrupole Focusing Lattice}

To reduce the beam size to less than that of the twoquadrupole focusing lattice, a lattice with more transverse focusing and a larger phase advance was explored. The five-quadrupole focusing lattice is shown in Figure 8.

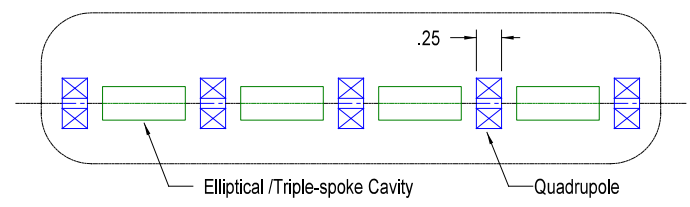

Figure 8: Layout of cryostat of the five-quadrupole focusing lattice.

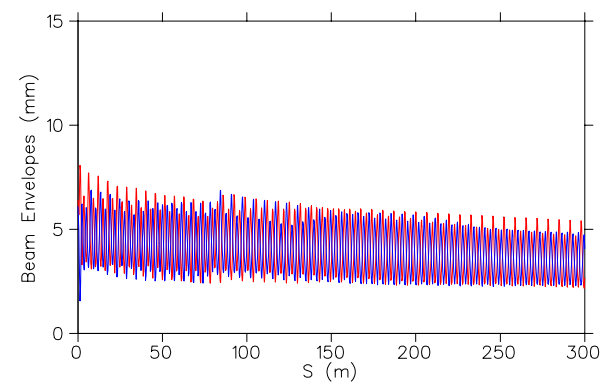

Figure 9: Multi-charge beam envelopes of ${ }^{238} \mathrm{U}$ in the fivequadrupole focusing lattice with Triple-spoke cavities.

The effective lengths of the quadrupole magnets remain $0.25 \mathrm{~m}$ with an aperture of $5 \mathrm{~cm}$. The maximum pole face magnetic field required for these quadrupole magnets to maintain each cryostat with a transverse phase advance of about $235^{\circ}$ is about $0.75 \mathrm{~T}$. Similarly, a short section with 
four quadrupole magnets is required to match the beam from the $2^{\text {nd }}$ charge-stripping chicane to the regular focusing lattice. Figure 9 shows the similar multi-charge beam envelopes for ${ }^{238} \mathrm{U}$ beam. The maximum beam size for all the three charge states is about $8 \mathrm{~mm}$. However, the additional quadrupoles increase the required cryostat length by about $20 \%$.

\section{MISALIGNMENT AND CORRECTIONS}

The lattice sensitivity to misalignment of focusing elements and cavities was investigated using DIMAD for all three transverse focusing lattices and for both elliptical and triple-spoke cavity types. The cavity choice has little impact on the lattice sensitivity based on our simulations. All accelerating structures and focusing elements were misaligned assuming a Gaussian distribution $( \pm 2 \sigma)$. The same correction scheme was used for all three lattices. A package in front of each cryostat consisting of a pair of horizontal and vertical orbit correctors and a beam position monitor (BPM) was assumed for the orbit correction scheme. A least-square-fit algorithm was used to obtain the horizontal and vertical corrector values that minimized the orbit distortions at all BPMs. Multi-charge beam simulations were then done to evaluate the impact on the lattice performance.

Figure 10 shows the misalignment simulation results for all three focusing lattices, considered showing a nearly linear dependence on focusing element misalignment. (The position errors for the cavities were fixed at $1.0 \mathrm{~mm}$.) The triple-spoke cavity will have a much smaller aperture with $3 \mathrm{~cm}$ or possibly $4 \mathrm{~cm}$, being considered, while the $805 \mathrm{MHz}$ 6-cell elliptical cavities have a much larger aperture $(7.7 \mathrm{~cm})$. To avoid uncontrollable beam halo formation due to nonlinear radial accelerating field dependence in the cavities, 1/3 of the cavity aperture was assumed to be an exclusion zone for the beam as shown in shaded areas in Figure 10.

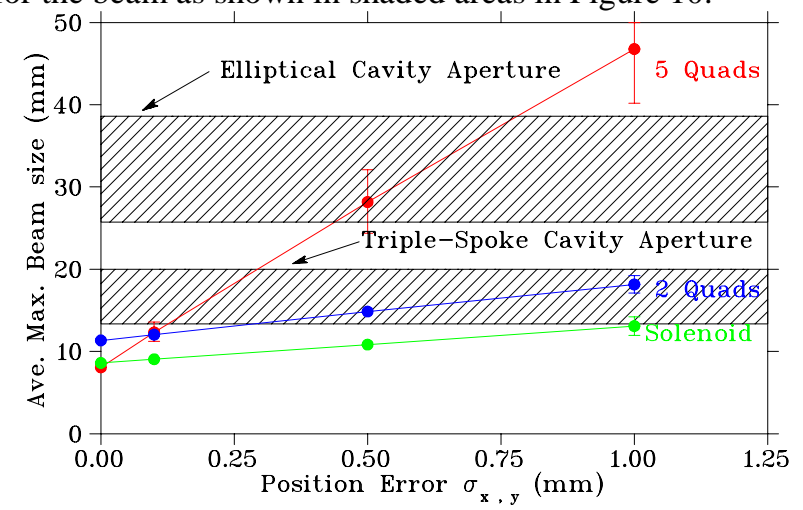

Figure 10: Average maximum beam sizes vs. misalignment errors for all three focusing lattices. The apertures of elliptical and triple-spoke cavities are also shown. The shaded areas are exclusion zones for each cavity type.

Due to the large phase advance and unfavorable ratio of correctors and BPMs vs. quadrupoles, the five-quadrupole focusing lattice is the most sensitive to the misalignment errors and will require unreasonable alignment requirements to maintain the beam inside the exclusion zones for both types of the cavities. The solenoid focusing lattice has the smallest maximum beam size, and with misalignment position errors of $1.0 \mathrm{~mm}$ for both the solenoids and cavities, is adequate for either cavity option. The 2-quadrupole focusing lattice has sensitivities to the misalignment errors similar to the solenoid focusing lattice, and would be adequate for the 6-cell elliptical cavity option. However with a reasonable misalignment tolerance, it will not be suitable for even a $4 \mathrm{~cm}$ aperture triple-spoke cavity option.

\section{CONCLUSION}

Both the $805 \mathrm{MHz}$ 6-cell elliptical and $322 \mathrm{MHz}$ triplespoke cavities would in principle provide the necessary acceleration. Due to its lower frequency and therefore longer effective accelerating gap, fewer cavities are needed for the $322 \mathrm{MHz}$ triple-spoke cavity option. However, the triple-spoke cavity will have a much smaller aperture, as the result, requires more expensive superconducting solenoid focusing lattice. No triple-spoke cavity has been built so far and its performance is untested. The $805 \mathrm{MHz}$ 6-cell elliptical cavities have a much larger aperture and have been successfully tested in 2002. A cost effective 2-Quadrupole focusing lattice (superconducting or room-temperature) with reasonable misalignment tolerances for both quadrupoles and cavities will be an adequate choice with the elliptical structure linac.

\section{REFERENCES}

[1] C.W. Leemann, "RIA Facility Project", Proc. of LINAC 2000, Monterey, CA, 2000.

[2] K.W. Shepard, "The RIA Driver Linac", Proc. of LINAC 2002, Gyeonju, Korea, 2002.

[3] D. Gorelov, T. Grimm, W. Hartung, F. Marti, H. Podlech, X. Wu and R.C. York, "Beam Dynamics Studies at NSCL of the RIA Superconducting Driver Linac", Proc. of EPAC 2002, Paris, France, 2002.

[4] W. Hartung, C.C. Compton, T.L. Grimm, R.C. York, G. Ciovati, P. Kneisel, "Status Report on Multi-Cell Superconducting Cavity Development for MediumVelocity Beams", these proceedings.

[5] D.V. Gorelov and P.N. Ostroumov, "Application of LANA Code for Design of Ion Linac" Proc. of EPAC 1996, Sitges, June 1996.

[6] R. Servranckx, K. Brown, L. Schachinger, and D. Douglas, "User's Guide to the Program Dimad", SLAC Report 285, UC-28, May 1985.

[7] T.L. Grimm, W. Hartung, M. Johnson, R.C. York, P. Kneisel, L. Turlington, "Cryomodule Design for the Rare Isotope Accelerator", these proceedings.

[8] X. Wu, D. Gorelov, T. Grimm, W. Hartung, F. Marti, and R.C. York, "The Misalignment and RF Error Analyses for the RIA Driver Linac", Proc. of LINAC 2002, Gyeonju, Korea, 2002. 